\title{
MAPPINGS OF FINITE DISTORTION AND ASYMMETRY OF DOMAINS
}

\section{Gioconda Moscariello, Antonia Passarelli di Napoli and Kai Rajala}

\author{
Università degli Studi di Napoli Federico II \\ Dipartimento di Matematica ed Applicazioni "R. Caccioppoli" \\ via Cintia, 80126 Napoli, Italy; gmoscari@unina.it \\ Università degli Studi di Napoli Federico II \\ Dipartimento di Matematica ed Applicazioni "R. Caccioppoli" \\ via Cintia, 80126 Napoli, Italy; antpassa@unina.it \\ University of Jyväskylä, Department of Mathematics and Statistics \\ P.O. Box 35, FI-40014 University of Jyväskylä, Finland; kai.i.rajala@jyu.fi
}

\begin{abstract}
We establish an anisotropic Fuglede inequality for images of balls under homeomorphisms with exponentially integrable distortion.
\end{abstract}

\section{Introduction}

In the last few years, the so-called quantitative isoperimetric inequalities have attracted a great interest (see for example $[6,3]$ and the references therein). In order to describe these results let us introduce, for any Borel set $E$ in $\mathbf{R}^{n}$, with $0<|E|<+\infty$, the isoperimetric deficit of $E$

$$
\delta(E)=\frac{P(E)}{n \omega_{n}^{\frac{1}{n}}|E|^{\frac{n-1}{n}}}-1=\frac{P(E)-P(r B)}{P(r B)},
$$

where $P$ is the perimeter (surface measure of the boundary if $E$ is smooth) and $r$ is the radius of the ball having the same volume of $E$, i.e. $|E|=r^{n} \omega_{n}$. Notice that $\delta(E)$ is non-negative by the isoperimetric inequality, and equals 0 if and only if $E$ is equivalent to a ball.

Fuglede [4] proved that if $E$ is a convex set with volume $\omega_{n}$, then

$$
\min _{x \in \mathbf{R}^{n}} \operatorname{dist}_{H}(E, x+B) \leq C(n) \delta(E)^{\alpha(n)}
$$

where $\operatorname{dist}_{H}(E, x+B)$ denotes the Hausdorff distance between the sets $E$ and $x+B$, and $\alpha(n)$ is a suitable exponent depending on the dimension $n$. When dealing with general non convex sets, one cannot expect the validity of inequalities like that proved by Fuglede, as can be seen by taking sets obtained by gluing thin long "tentacles" to the unit ball. Fuglede's result has been generalized to the class of John domains in [18], see also [5].

Fusco, Maggi and Pratelli [6] gave a sharp estimate for the Fraenkel asymmetry (see (1.1)) of a set $E$ in terms of its isoperimetric deficit, proving a conjecture by Hall

doi:10.5186/aasfm.2013.3803

2010 Mathematics Subject Classification: Primary 30C65, 46E35.

Key words: Mappings of finite distortion, isoperimetric inequality.

Rajala was supported by the Academy of Finland. Part of this research was done when Rajala was visiting the University of Naples Federico II. He thanks the department for its hospitality. 
[9]. Very recently, an anisotropic version of the result in [6] was established by Figalli, Maggi and Pratelli in [3]. More precisely, given a convex set $E, 0<|E|<+\infty$, containing the origin, and an open set $F, 0<|F|<+\infty$, with smooth boundary $\partial F$ oriented by its unit outer normal $\nu_{F}$, the anisotropic perimeter of $F$ is defined as

$$
P_{E}(F)=\int_{\partial F}\left\|\nu_{F}(x)\right\|_{*} d \mathcal{H}^{n-1}(x)
$$

where

$$
\left\|\nu_{F}\right\|_{*}=\sup \left\{y \cdot \nu_{F}: y \in E\right\} .
$$

Note that, when $E$ is the unit ball $B$, the perimeter $P_{E}(F)$ coincides with the usual notion of Euclidean perimeter. It is possible to extend the definition of anisotropic perimeter also to non-smooth sets, by using the notion of reduced boundary (see [1] or [3, Section 2.1]). Then, let us introduce the isoperimetric deficit of $F$, setting

$$
\delta_{E}(F)=\frac{P_{E}(F)}{n|E|^{\frac{1}{n}}|F|^{\frac{1}{n^{\prime}}}}-1,
$$

and the relative asymmetry of $E$ and $F$ as

$$
A(E, F)=\inf _{b \in \mathbf{R}^{n}} \frac{|E \backslash(b+\kappa F)|}{|E|}, \quad \kappa=\left(\frac{|E|}{|F|}\right)^{1 / n},
$$

where $\kappa F=\{\kappa y: y \in F\}$. Notice that $A(E, F)=A(F, E), A(\lambda E, \mu F)=A(E, F)$ for every $\lambda$ and $\mu>0$ and that if $E$ is the unit ball, the relative asymmetry coincides with the Fraenkel asymmetry of $F$.

In [3] it has been proved that, if $F$ is a measurable set with finite perimeter and finite positive measure, then $A(F, E)$ can be controlled by the isoperimetric deficit. More precisely, $A(F, E) \leq C \sqrt{\delta_{E}(F)}$ for a constant $C$ depending only on $n$.

The aim of this paper is to prove an anisotropic version of the Fuglede type inequality in higher dimension, restricting ourselves to the class of domains which are images of the unit ball under global homeomorphisms with exponentially integrable distortion. We recall that $f: \Omega \subset \mathbf{R}^{n} \rightarrow \mathbf{R}^{n}$ is a mapping of finite distortion, if $f \in$ $W_{\text {loc }}^{1,1}\left(\Omega, \mathbf{R}^{n}\right)$, the Jacobian determinant $J_{f} \in L_{\mathrm{loc}}^{1}(\Omega)$, and if there is a measurable, almost everywhere finite function $K_{f}$ such that

$$
|D f(x)|^{n} \leq K_{f}(x) J_{f}(x) \text { for almost every } x \in \Omega .
$$

If $E$ and $F$ are as above, we recall that the Hausdorff distance between $\partial E$ and $\partial F$ is

$$
\operatorname{dist}_{H}(\partial E, \partial F)=\max \left\{\sup _{x \in \partial E} \inf _{y \in \partial F}|x-y|, \sup _{y \in \partial F} \inf _{x \in \partial E}|x-y|\right\} .
$$

We define the relative distance dist $_{\mathrm{rel}}(\partial E, \partial F)$,

$$
\operatorname{dist}_{\text {rel }}(\partial E, \partial F)=\inf _{b \in \mathbf{R}^{n}} \frac{\operatorname{dist}_{H}(\partial E, b+\kappa \partial F)}{|E|^{1 / n}},
$$

where $\kappa$ is the constant in (1.1). Notice that the relative distance is also scaling and translation invariant.

In [17], it has been proved that the Hausdorff distance between the images of the unit ball under homeomorphisms with exponentially integrable distortion and the unit ball can be controlled by their Fraenkel asymmetry. Our main result states that we can control the relative distance between the images of the unit sphere 
under two homeomorphisms with exponentially integrable distortion with the relative asymmetry of the images of the unit ball. More precisely, we have

Theorem 1.1. Let $f: B_{2} \rightarrow f B_{2}$ and $g: B_{2} \rightarrow g B_{2}$ be homeomorphisms of finite distortion satisfying

$$
\int_{B_{2}} \exp \left(\mu K_{f}(x)\right)+\exp \left(\mu K_{g}(x)\right) \mathrm{d} x=\mathcal{K}<\infty
$$

for some $\mu$ and $\mathcal{K}>0$. Then

$$
\operatorname{dist}_{\text {rel }}\left(f S_{1}, g S_{1}\right)^{n+n^{2} / \mu} \leq C(n, \mu, \mathcal{K}) A\left(f B_{1}, g B_{1}\right) .
$$

As a consequence of Theorem 1.1, we obtain that the relative distance between the image of the unit sphere under a homeomorphism with exponentially integrable distortion and the boundary of a convex set can be estimated with the relative asymmetry between the convex set and the image of the unit ball. Namely, we have

Theorem 1.2. Let $f: B_{2} \rightarrow f B_{2}$ be a homeomorphism of finite distortion satisfying

$$
\int_{B_{2}} \exp \left(\mu K_{f}(x)\right) \mathrm{d} x=\mathcal{K}<\infty
$$

for some $\mu$ and $\mathcal{K}>0$, and let $E$ be a convex domain, $B_{1} \subset E \subset B_{\Lambda}$. Then

$$
\operatorname{dist}_{\text {rel }}\left(f S_{1}, \partial E\right)^{n+n^{2} / \mu} \leq C(n, \mu, \mathcal{K}, \Lambda) A\left(f B_{1}, E\right) \leq C(n, \mu, \mathcal{K}, \Lambda) \sqrt{\delta_{E}\left(f B_{1}\right)} .
$$

Notice that the second inequality in the previous theorem follows by the result in [3]. Let $E$ be a convex set with finite positive Lebesgue measure and let $B_{1} \subset E \subset B_{\Lambda}$. We notice that the estimate of Theorem 1.2 could blow up when $\Lambda$ goes to infinity. In fact, we can take $f$ to be the identity mapping and a sequence of convex sets $E_{j}$ converging to a line segment. Then the right hand side of (1.3) tends to infinity while the left hand side remains bounded.

Theorems 1.1 and 1.2 do not hold in general if $f$ is only defined on $B_{1}$. Indeed, for every $n \geq 2$ there exist $K \geq 1$ and a sequence of $K$-quasiconformal homeomorphisms $f_{j}: B_{1} \rightarrow \Omega_{j}$ such that $\Omega_{j}$ is the union of $B_{1}$ and a truncated cone with vertex $2 e_{1}$ and opening angle $1 / j$. This can be seen using the fact that truncated cones with different opening angles smaller than $\pi / 2$ are quasiconformally equivalent with distortion independent of the angles, see [8]. The exponent that appears in estimates (1.2) and (1.3) cannot be improved, except for the constant $n^{2}$, as shown in the example at Theorem 1.2 of [17].

We would like to mention that Theorem 1.2 gives an extension of the main Theorem 1.1 in [17]. Indeed, this is implied by Theorem 1.2, in case $E$ is assumed to be a ball. The approach we use to prove Theorems 1.1 and 1.2 is quite similar to that used in [17] earlier. However, we are able to prove much more general results because of our new observation that the approach can be applied to give estimates for the distances of rather general domains (in particular without assuming that one of them is a ball).

Quantitative isoperimetric inequalities have been applied to prove new distortion estimates for quasiconformal maps with small distortion, see $[15,16]$. More generally, we expect that Theorems 1.1 and 1.2 can be applied to give estimates for quasiconformal maps whose distortions are suitably controlled using a convex domain $E$. 


\section{Notations and preliminary results}

We shall denote a ball in $\mathbf{R}^{n}$ with center $x$ and radius $r$ by $B_{r}(x)$, while when the ball is centered at the origin we shall omit the indication of the center, i.e. $B_{r}=B_{r}(0)$. The corresponding notations for spheres will be $S_{r}(x)$ and $S_{r}$.

We shall denote by $|D f|$ the operator norm of the differential matrix and by $(D f)^{\sharp}$ the adjugate of $D f$ which is defined by the formula

$$
D f \cdot(D f)^{\sharp}=\mathbf{I} \cdot J_{f},
$$

where, as usual, $J_{f}=\operatorname{det} D f$ and $\mathbf{I}$ is the identity matrix.

Recall that a homeomorphism $f \in W_{\text {loc }}^{1,1}\left(\Omega, \mathbf{R}^{n}\right)$ has finite outer distortion if its Jacobian $J_{f}$ is strictly positive a.e. on the set where $|D f| \neq 0$. In case $J_{f}(x) \geq 0$ a.e., we define its outer distortion function as

$$
K_{f}(x)= \begin{cases}\frac{|D f(x)|^{n}}{J_{f}(x)} & \text { for } J_{f}(x)>0 \\ 1 & \text { otherwise. }\end{cases}
$$

We note, for a homeomorphism with finite distortion, the following relation:

$$
\left|\left(D f^{-1}\right)^{\sharp}(y)\right|=K_{f}\left(f^{-1}(y)\right)^{\frac{1}{n}} J_{f^{-1}}(y)^{\frac{n-1}{n}} \quad \text { a.e. } y \in f(\Omega)
$$

(we refer to [11] for an exhaustive treatment of the mappings with finite distortion). We shall use following result concerning the modulus of continuity of a homeomorphism with exponentially integrable distortion.

Theorem 2.1. [14] Let $f$ be as in Theorem 1.1. If $x$ and $y \in B_{5 / 4}$, then

$$
|f(x)-f(y)| \leq \frac{C(n, \mu, \mathcal{K})}{\log ^{\mu / n} \frac{1}{|x-y|}}\left|f B_{1}\right|^{1 / n} .
$$

Moreover, we have the following distortion estimate.

Lemma 2.2. [17] Let $f$ be as in Theorem 1.1. If $B_{t}(x) \subset B_{5 / 4}$, then there exists a constant $C=C(n, \mu, \mathcal{K})$ such that

$$
\frac{\max _{y \in S_{3 / 2}}|f(x)-f(y)|}{\min _{y \in S_{t}(x)}|f(x)-f(y)|} \leq \exp \left(C(n, \mu, \mathcal{K}) t^{-\frac{1}{n-\frac{3}{2}}}\right) .
$$

We will use the following consequence of the previous results.

Lemma 2.3. Let $f$ be as above and assume $f\left(e_{1}\right)=0$ and $\left|f B_{1}\right|=\omega_{n}$. Then there exists $m_{0}>0$, depending only on $n, \mu$, and $\mathcal{K}$, such that for every $0<t<m_{0}$,

$$
B_{s_{t}}\left(e_{1}\right) \subset f^{-1} B_{t} \subset B_{1 / 10}\left(e_{1}\right) .
$$

Here $s_{t}$ satisfies

$$
t=\frac{C(n, \mu, \mathcal{K})}{\log ^{\mu / n} \frac{1}{s_{t}}},
$$

where $C(n, \mu, \mathcal{K})$ is the constant in Theorem 2.1. Moreover,

$$
\left|B_{1} \cap f^{-1} B_{t}\right|^{(n-1) / n} \leq C(n) \mathcal{H}^{n-1}\left(B_{1} \cap f^{-1} S_{t}\right) .
$$


Proof. The first inclusion in (2.4) follows directly from Theorem 2.1. Also, under the assumption $\left|f B_{1}\right|=\omega_{n}$, there exists $b \in S_{3 / 2}$ such that $\left|f\left(e_{1}\right)-f(b)\right|=|f(b)| \geq 1$. Lemma 2.2 with $x=e_{1}$ yields

$$
\frac{1}{\min _{y \in S_{1 / 10}\left(e_{1}\right)}\left|f(y)-f\left(e_{1}\right)\right|} \leq \frac{\max _{b \in S_{3 / 2}}|f(b)|}{\min _{y \in S_{1 / 10}\left(e_{1}\right)}|f(y)|} \leq \varphi(n, \mu, \mathcal{K}) .
$$

The second inclusion follows once $m_{0}$ is chosen to be small enough depending only on $n, \mu$, and $\mathcal{K}$.

To prove (2.5), we notice that the second inclusion in (2.4) guarantees that

$$
\left|B_{1} \cap f^{-1} B_{t}\right| \leq\left|B_{1} \backslash f^{-1} B_{t}\right| \text {. }
$$

Therefore, (2.5) follows by the following relative isoperimetric inequality in $B_{1}$ (see $[1,(3.43)])$ :

$$
\min \left\{\left|B_{1} \cap f^{-1} B_{t}\right|^{(n-1) / n},\left|B_{1} \backslash f^{-1} B_{t}\right|^{(n-1) / n}\right\} \leq C(n) \mathcal{H}^{n-1}\left(B_{1} \cap f^{-1} S_{t}\right) .
$$

\section{Proof of Theorem 1.1}

We assume that $A\left(f B_{1}, g B_{1}\right)>0$. Since the distortions $K_{f}$ and $K_{g}$ are not affected by postcompositions with affine maps, we may assume that $\left|f B_{1}\right|=\left|g B_{1}\right|=$ $\omega_{n}$ and

$$
A\left(f B_{1}, g B_{1}\right)=\omega_{n}^{-1}\left|f B_{1} \backslash g B_{1}\right|=\omega_{n}^{-1}\left|g B_{1} \backslash f B_{1}\right| .
$$

We denote $m=\operatorname{dist}_{H}\left(f S_{1}, g S_{1}\right)$. In order to prove the theorem, we need to show that

$$
m^{n+n^{2} / \mu} \leq C(n, \mu, \mathcal{K})\left|f B_{1} \backslash g B_{1}\right| .
$$

Now either there exists a point $y_{0} \in f S_{1}$ such that

$$
\operatorname{dist}_{H}\left(y_{0}, g S_{1}\right)=m \text {, }
$$

or a point $z_{0} \in g S_{1}$ such that

$$
\operatorname{dist}_{H}\left(z_{0}, f S_{1}\right)=m .
$$

From now on we assume that (3.1) holds, otherwise we change the roles of $f$ and g. By precomposing $f$ with a rotation and postcomposing with a translation, if necessary, we may assume that $y_{0}=0$ and $f^{-1}\left(y_{0}\right)=e_{1}$.

Since $\operatorname{dist}_{H}\left(0, g S_{1}\right)=m$, we have that either

$$
B_{m} \cap f B_{1} \subset \mathbf{R}^{n} \backslash g B_{1},
$$

or

$$
B_{m} \backslash f B_{1} \subset g B_{1},
$$

depending on whether or not $0 \in \mathbf{R}^{n} \backslash g B_{1}$. In the first case we have

$$
\left|f B_{1} \backslash g B_{1}\right| \geq\left|B_{m} \cap f B_{1} \backslash g B_{1}\right|=\left|B_{m} \cap f B_{1}\right|,
$$

so in order to prove the theorem it suffices to show that

$$
m^{n+n^{2} / \mu} \leq C(n, \mu, \mathcal{K})\left|B_{m} \cap f B_{1}\right| .
$$

In the second case

$$
\left|g B_{1} \backslash f B_{1}\right| \geq\left|B_{m} \backslash f B_{1}\right|,
$$

so in this case the theorem follows if

$$
m^{n+n^{2} / \mu} \leq C(n, \mu, \mathcal{K})\left|B_{m} \backslash f B_{1}\right| .
$$


The proofs of estimates (3.3) and (3.4) are very similar, and therefore we only give the proof of (3.3).

Proof of (3.3). We first assume that $m \leq m_{0}$, where $m_{0}$ is as in Lemma 2.3. We choose $t_{0}=m / 2$ and an increasing sequence $\left(t_{j}\right)_{j=1}^{k}$ of radii inductively such that if

$$
\left|f^{-1}\left(B_{m}\right) \cap B_{1}\right|>2\left|f^{-1}\left(B_{t_{j-1}}\right) \cap B_{1}\right|
$$

then we choose $m / 2<t_{j}<m$ such that

$$
\left|f^{-1}\left(B_{t_{j}}\right) \cap B_{1}\right|=2\left|f^{-1}\left(B_{t_{j-1}}\right) \cap B_{1}\right|,
$$

otherwise $j=k$ and we choose $t_{k}=m$. We claim that

$$
k \leq C(n) \log \frac{1}{s_{m / 2}} \leq C(n, \mu, \mathcal{K}) m^{-n / \mu} .
$$

Here $s_{m / 2}$ is as in Lemma 2.3. The second inequality in (3.6) follows from Lemma 2.3. In order to prove the first inequality we use the definition of $\left(t_{j}\right)$ to write (note that $\left.t_{k}=m\right)$

$$
2^{k-1} \leq \prod_{j=1}^{k} \frac{\left|f^{-1}\left(B_{t_{j}}\right) \cap B_{1}\right|}{\left|f^{-1}\left(B_{t_{j-1}}\right) \cap B_{1}\right|}=\frac{\left|f^{-1}\left(B_{m}\right) \cap B_{1}\right|}{\left|f^{-1}\left(B_{m / 2}\right) \cap B_{1}\right|}
$$

Since

$$
B_{s_{m / 2}}\left(e_{1}\right) \subset f^{-1}\left(B_{m / 2}\right) \text { and } f^{-1}\left(B_{m}\right) \subset B_{1 / 10}\left(e_{1}\right),
$$

by Lemma 2.3 , the right term in (3.7) can be estimated by $C(n) s_{m / 2}^{-n}$. Taking logarithms gives the first inequality in (3.6).

By [2] (see also [7, 10, 13]), $f^{-1} \in W^{1, n}\left(f B_{2}, \mathbf{R}^{n}\right)$. Therefore,

$$
\mathcal{H}^{n-1}\left(f^{-1}\left(S_{t} \cap f B_{1}\right)\right) \leq \int_{S_{t} \cap f B_{1}}\left|\left(D f^{-1}\right)^{\sharp}(y)\right| \mathrm{d} \mathcal{H}^{n-1}(y)
$$

for almost every $m / 2<t<m$. Indeed, recall that the change of variables formula holds for $h: G \subset \mathbf{R}^{k} \rightarrow \mathbf{R}^{n}, k \leq n$, whenever $h \in W^{1, p}$ for some $p>k$, see [12]. We conclude that (3.8) holds since the restriction of our $f^{-1}$ to (the relative components of) $S_{t} \cap f B_{1}$ belongs to $W^{1, n}$ for almost every $m / 2<t<m$.

Let $V_{t}=f^{-1}\left(B_{t}\right) \cap B_{1}$ and $V_{j}=V_{t_{j}}$. We integrate both sides of (3.8) over the interval $\left(t_{j-1}, t_{j}\right), j=1, \ldots, k$. By the relative isoperimetric inequality $(2.5)$, and our choice (3.5) of the radii $t_{j}$, the left integral is estimated from below by

$$
C(n)\left(t_{j}-t_{j-1}\right)\left|V_{j}\right|^{(n-1) / n} \text {. }
$$

Using the equality at (2.3), Hölder's inequality and the area formula, the right integral is estimated from above as follows:

$$
\begin{aligned}
\int_{f B_{1} \cap B_{t_{j}} \backslash B_{t_{j-1}}}\left|\left(D f^{-1}\right)^{\sharp}(y)\right| \mathrm{d} y & =\int_{f B_{1} \cap B_{t_{j}} \backslash B_{t_{j-1}}} K_{f}\left(f^{-1}(y)\right)^{1 / n} J_{f^{-1}}(y)^{(n-1) / n} \mathrm{~d} y \\
& \leq\left|f B_{1} \cap B_{t_{j}} \backslash B_{t_{j-1}}\right|^{1 / n}\left(\int_{V_{j}} K_{f}(x)^{1 /(n-1)} \mathrm{d} x\right)^{(n-1) / n} .
\end{aligned}
$$

We denote $\left|f B_{1} \cap B_{t_{j}} \backslash B_{t_{j-1}}\right|$ by $q_{j}$. Combining the estimates and taking the measure of $V_{j}$ to the right yields

$$
\left(t_{j}-t_{j-1}\right) \leq C q_{j}^{1 / n}\left(\left|V_{j}\right|^{-1} \int_{V_{j}} K_{f}(x)^{1 /(n-1)} \mathrm{d} x\right)^{(n-1) / n},
$$


where $C$ depends only on $n$. Applying Jensen's inequality to the convex function $t \mapsto \exp \left(\mu t^{n-1}\right)$, and using the integrability assumption on $K_{f}$, gives

$$
\begin{aligned}
\left|V_{j}\right|^{-1} \int_{V_{j}} K_{f}(x)^{1 /(n-1)} \mathrm{d} x & \leq \mu^{-1}\left(\log \left(\left|V_{j}\right|^{-1} \int_{V_{j}} \exp \left(\mu K_{f}(x)\right) \mathrm{d} x\right)\right)^{1 /(n-1)} \\
& \leq \mu^{-1}\left(\log \left(\mathcal{K}\left|V_{j}\right|^{-1}\right)\right)^{1 /(n-1)}
\end{aligned}
$$

Since $\left|V_{j}\right| \geq C(n) s_{m / 2}^{n}$, where $s_{m / 2}$ is as in Lemma 2.3, the second inequality in (3.6) yields

$$
\left(\log \left|V_{j}\right|^{-1}\right)^{1 /(n-1)} \leq C(n, \mu, \mathcal{K}) m^{-n /(\mu(n-1))} .
$$

Combining with (3.9) and (3.10) gives

$$
t_{j}-t_{j-1} \leq C q_{j}^{1 / n} m^{-1 / \mu}
$$

Finally, we add over $j$ and use the Cauchy-Schwarz inequality:

$$
\frac{m}{2}=\sum_{j=1}^{k} t_{j}-t_{j-1} \leq C m^{-1 / \mu} \sum_{j=1}^{k} q_{j}^{1 / n} \leq C m^{-1 / \mu} k^{(n-1) / n}\left(\sum_{j=1}^{k} q_{j}\right)^{1 / n} .
$$

Since

$$
\sum_{j=1}^{k} q_{j} \leq\left|B_{m} \cap f B_{1}\right|
$$

using estimates (3.11) and (3.6), we get

$$
m \leq C(n, \mu, \mathcal{K})\left|B_{m} \cap f B_{1}\right|^{1 / n} m^{-n / \mu},
$$

which implies (3.3).

We next assume that $m \geq m_{0}$. By applying the previous argument with $m=m_{0}$, we see that

$$
\left|B_{m} \cap f B_{1}\right| \geq\left|B_{m_{0}} \cap f B_{1}\right| \geq C_{1}(n, \mu, \mathcal{K}) .
$$

On the other hand, Lemma 2.2 shows that we always have

$$
m \leq \operatorname{diam} f S_{1} \leq C_{2}(n, \mu, \mathcal{K})\left|f B_{1}\right|=C_{3}(n, \mu, \mathcal{K}) .
$$

Combining (3.12) and (3.13) gives (3.3). The proof is complete.

\section{Proof of Theorem 1.2}

We use the following result of Gehring and Väisälä [8, Theorem 5.2] (they only consider the case $n=3$, but the proof extends to all dimensions $n \geq 2$ ).

Theorem 4.1. Let $B_{1} \subset E \subset B_{\Lambda}$ be a convex domain. Then there exists a $K$-quasiconformal mapping $g: \mathbf{R}^{n} \rightarrow \mathbf{R}^{n}$, where $K$ depends only on $n$ and $\Lambda$, such that $g B_{1}=E$.

Proof of Theorem 1.2. We shall apply Theorems 1.1 and 4.1. By scaling invariance, we may assume that $\left|f B_{1}\right|=|E|=\omega_{n}$ and

$$
A\left(f B_{1}, E\right)=\omega_{n}^{-1}\left|f B_{1} \backslash E\right| .
$$


By Lemmas 2.2 and 2.3, there exist $x \in \mathbf{R}^{n}$ and $t>1$, depending only on $n, \mu$, and $\mathcal{K}$, such that

$$
B_{t^{-1}}(x) \subset f B_{1} \subset B_{t}(x) .
$$

Let $\Lambda$ be the smallest constant for which there exist $x \in \mathbf{R}^{n}$ and $R>0$ such that

$$
B_{R}(x) \subset E \subset B_{\Lambda R}(x) .
$$

Then, if $\Lambda$ is large enough depending on $t$, the convexity of $E$ implies that $A\left(f B_{1}, E\right) \geq$ $1 / 100$. We have $R<1$ because $|E|=\omega_{n}$, and so

$$
\operatorname{dist}_{\text {rel }}\left(f S_{1}, \partial E\right) \leq \operatorname{diam} f S_{1}+\operatorname{diam} \partial E \leq C(n, \mu, \mathcal{K})+\Lambda .
$$

Therefore,

$$
\operatorname{dist}_{\text {rel }}\left(f S_{1}, \partial E\right) \leq C(n, \mu, \mathcal{K}, \Lambda) A\left(f B_{1}, E\right)
$$

when $\Lambda \geq \Lambda_{0}(n, \mu, \mathcal{K})$.

Now let $\Lambda \leq \Lambda_{0}(n, \mu, \mathcal{K})$. An application of Theorem 4.1 gives a $K(n, \mu, \mathcal{K})$ quasiconformal homeomorphism $g: B_{2} \rightarrow g B_{2}$ such that $g B_{1}=E$. We can now apply Theorem 1.1 to $f$ and $g$, since

$$
\int_{B_{2}} \exp \left(\mu K_{g}(x)\right) \mathrm{d} x \leq C(n, \mu, \mathcal{K}) .
$$

This gives the first inequality at (1.3). The second inequality follows from $[3$, Theorem $1.1]$.

\section{References}

[1] Ambrosio, L., N. Fusco, and D. Pallara: Functions of bounded variation and free discontinuity problems. - Oxford Math. Monogr., Oxford Univ. Press, 2000.

[2] Csörnyei, M., S. Hencl, and J. Malý: Homeomorphisms in the Sobolev space $W^{1, n-1}$. J. Reine Angew. Math. 644, 2010, 221-235.

[3] Figalli, A., F. Maggi, and A. Pratelli: A mass tranportation approach to quantitative isoperimetric inequalities. - Invent. Math. 182:1, 2010, 167-211.

[4] Fuglede, B.: Stability in the isoperimetric problem for convex or nearly spherical domains in $\mathbf{R}^{n}$. - Trans. Amer. Math. Soc. 314:2, 1989, 619-638.

[5] Fusco, N., M. S. Gelli, and G. Pisante: On a Bonnesen type inequality involving the spherical deviation. - J. Math. Pures Appl. (to appear), doi:10.1016/j.matpur.2012.05.006.

[6] Fusco, N., F. Maggi, and A. Pratelli: The sharp quantitative isoperimetric inequality. Ann. of Math. (2) 168:3, 2008, 941-980.

[7] Fusco, N., G. Moscariello, and C. Sbordone: The limit of $W^{1,1}$ homeomorphisms with finite distortion. - Calc. Var. Partial Differential Equations 33, 2008, 377-390.

[8] Gehring, F. W., and J. VÄIsÄLÄ: The coefficients of quasiconformality of domains in space. - Acta Math. 114, 1965, 1-70.

[9] Hall, R. R.: A quantitative isoperimetric inequality in $n$-dimensional space. - J. Reine Angew. Math. 428, 1992, 161-176.

[10] Hencl, S., G. Moscariello, A. Passarelli di Napoli, and C. Sbordone: Bi-Sobolev mappings and elliptic equations in the plane. - J. Math. Anal. Appl. 355, 2009, 22-32.

[11] Iwaniec, T., and G. Martin: Geometric function theory and non-linear analysis. - Oxford Univ. Press, 2001. 
[12] Marcus, M., and V. J. Mizel: Transformations by functions in Sobolev spaces and lower semicontinuity for parametric variational problems. - Bull. Amer. Math. Soc. 79, 1973, 790795.

[13] Moscariello, G., and A. Passarelli di Napoli: The regularity of the inverses of Sobolev homeomorphisms. - Submitted.

[14] Onninen, J., and X. Zhong: A note on mappings of finite distortion: the sharp modulus of continuity. - Michigan Math. J. 53:2, 2005, 329-335.

[15] Rajala, K.: The local homeomorphism property of spatial quasiregular mappings with distortion close to one. - Geom. Funct. Anal. 15:5, 2005, 1100-1127.

[16] RAJALA, K.: Distortion of quasiconformal and quasiregular mappings at extremal points. Michigan Math. J. 53:2, 2005, 447-458.

[17] Rajala, K.: Quantitative isoperimetric inequalities and homeomorphisms with finite distortion. - Ann. Sc. Norm. Super. Pisa Cl. Sci. (5) 11:1, 2012, 177-196.

[18] Rajala, K., and X. Zhong: Bonnesen's inequality for John domains in $\mathbf{R}^{n}$. - J. Funct. Anal. (to appear).

Received 4 June $2012 \bullet$ Accepted 2 July 2012 